\title{
Comparison of various insulin resistance surrogates on prognostic prediction and stratification following percutaneous coronary intervention in patients with and without type 2 diabetes mellitus
}

\author{
Qi Zhao, Yu-Jing Cheng, Ying-Kai Xu, Zi-Wei Zhao, Chi Liu, Tie-Nan Sun and Yu-Jie Zhou* (1)
}

\begin{abstract}
Background: Insulin resistance (IR), evaluation of which is difficult and complex, is closely associated with cardiovascular disease. Recently, various IR surrogates have been proposed and proved to be highly correlated with IR assessed by the gold standard. It remains indistinct whether different IR surrogates perform equivalently on prognostic prediction and stratification following percutaneous coronary intervention (PCI) in non-ST-segment elevation acute coronary syndrome (NSTE-ACS) patients with and without type 2 diabetes mellitus (T2DM).

Methods: The present study recruited patients who were diagnosed with NSTE-ACS and successfully underwent PCI. IR surrogates evaluated in the current study included triglyceride-glucose (TyG) index, visceral adiposity index, Chinese visceral adiposity index, lipid accumulation product, and triglyceride-to-high density lipoprotein cholesterol ratio, calculations of which were conformed to previous studies. The observational endpoint was defined as the major adverse cardiovascular and cerebrovascular events (MACCE), including cardiac death, non-fatal myocardial infarction, and non-fatal ischemic stroke.
\end{abstract}

Results: 2107 patients (60.02 \pm 9.03 years, $28.0 \%$ female) were ultimately enrolled in the present study. A total of 187 (8.9\%) MACCEs were documented during the 24-month follow-up. Despite regarding the lower median as reference [hazard ratio (HR) 3.805, 95\% confidence interval (CI) 2.581-5.608, $\mathrm{P}<0.001$ ] or evaluating 1 normalized unit increase (HR 1.847, 95\% Cl 1.564-2.181, P<0.001), the TyG index remained the strongest risk predictor for MACCE, independent of confounding factors. The TyG index showed the most powerful diagnostic value for MACCE with the highest area under the receiver operating characteristic curve of 0.715 . The addition of the TyG index, compared with other IR surrogates, exhibited the maximum enhancement on risk stratification for MACCE on the basis of a baseline model (Harrell's C-index: 0.708 for baseline model vs. 0.758 for baseline model + TyG index, $\mathrm{P}<0.001$; continuous net reclassification improvement: $0.255, \mathrm{P}<0.001$; integrated discrimination improvement: $0.033, \mathrm{P}<0.001)$. The results were consistent in subgroup analysis where similar analyses were performed in patients with and without T2DM, respectively.

\footnotetext{
*Correspondence: azzyj12@163.com

Department of Cardiology, Beijing Anzhen Hospital, Capital Medical University, Beijing Institute of Heart Lung and Blood Vessel Disease, Beijing Key Laboratory of Precision Medicine of Coronary Atherosclerotic Disease, Clinical Center for Coronary Heart Disease, Capital Medical University, Beijing 100029, China
} permits use, sharing, adaptation, distribution and reproduction in any medium or format, as long as you give appropriate credit to the original author(s) and the source, provide a link to the Creative Commons licence, and indicate if changes were made. The images or other third party material in this article are included in the article's Creative Commons licence, unless indicated otherwise in a credit line to the material. If material is not included in the article's Creative Commons licence and your intended use is not permitted by statutory regulation or exceeds the permitted use, you will need to obtain permission directly from the copyright holder. To view a copy of this licence, visit http://creativecommons.org/licenses/by/4.0/. The Creative Commons Public Domain Dedication waiver (http://creativeco mmons.org/publicdomain/zero/1.0/) applies to the data made available in this article, unless otherwise stated in a credit line to the data. 
Conclusion: The TyG index, which is most strongly associated with the risk of MACCE, can be served as the most valuable IR surrogate for risk prediction and stratification in NSTE-ACS patients receiving PCI, with and without T2DM.

Keywords: Insulin resistance surrogates, Type 2 diabetes mellitus, Non-ST-segment elevation acute coronary syndrome, Percutaneous coronary intervention, Major adverse cardiac and cerebrovascular events

\section{Background}

Insulin resistance (IR), the most important pathogenesis for type 2 diabetes mellitus (T2DM) and metabolic syndrome, has been demonstrated to be closely related to the occurrence, progression, and prognosis of atherosclerotic cardiovascular disease (ASCVD), regardless of the presence of diabetes mellitus [1-6]. Therefore, there is undisputedly a demand for precise and prompt quantification of IR, with the aim of early identification of patients at high risk of ASCVD, assessment of disease progression, and risk stratification for adverse outcomes.

The hyperinsulinaemic-euglycaemic (HIEG) clamp, which is the gold standard technique for the evaluation of IR, has been demonstrated to be closely associated with ASCVD by previous studies [7, 8]. However, the defects of operational complexity, time consumption, and expensiveness confined it from extensive clinical application. It has been revealed that IR usually manifests as hyperglycemia, hyperinsulinemia, dyslipidemia, and central obesity (especially increased visceral fat) [6,9]. Based on the characteristics mentioned above, various surrogate markers calculated from common laboratory and anthropometric parameters, for example, triglyceride-glucose index (TyG index), visceral adiposity index (VAI), Chinese visceral adiposity index (CVAI), lipid accumulation product (LAP), and triglyceride-to-high density lipoprotein cholesterol ratio (TG/HDL-C), have been established to alternatively evaluate the extent of IR and shown to be closely correlated with HIEG clamp [10-16]. The level of IR assessed by these surrogates has been shown in numerous studies to be significantly associated with the risk of prediabetes/diabetes, atherosclerosis, ASCVD, and adverse prognosis [17-26].

At present, comprehensive evaluation and comparison of various IR surrogates for risk prediction and stratification of adverse prognosis after percutaneous coronary intervention (PCI) in non-ST-segment elevation acute coronary syndrome (NSTE-ACS) patients with and without T2DM are still inadequate. Therefore, the current study was designed to explore the underlying relationship of various IR surrogates with adverse prognosis in this selected high-risk population, and determine the superiority among them on prognostic prediction and stratification.

\section{Methods}

\section{Study population}

As a single-center observational cohort study, we screened patients admitted for nonemergent coronary procedures at Beijing Anzhen Hospital, Capital Medical University, between June 1st, 2018 and June 1st, 2019. The inclusion criteria were as follows: (1) age $\geq 18$ years; (2) diagnosed with NSTE-ACS [non-ST-segment elevation myocardial infarction (NSTEMI) or unstable angina (UA)], diagnostic criteria of which were referred to relevant guidelines [27]; (3) successfully underwent PCI, which was defined as residual stenosis of the target lesion $<30 \%$ by visual examination or quantitative assessment, and the absence of serious adverse cardiac events during hospitalization. Patients with missing baseline data, previous history of coronary artery bypass grafting, suspected familial hypertriglyceridemia, and/or other exclusion criteria were excluded (details shown in Fig. 1).

The study protocol was endorsed by the Clinical Research Ethics Committee of Beijing Anzhen Hospital, Capital Medical University. All subjects were informed and agreed to participate in the present study.

\section{Data collection and definitions}

Demographic, anthropometric, laboratory, medical and procedural information was acquired by referring to the electronic medical record management system of Beijing Anzhen Hospital and then entered into an established database by trained personnel who was blinded to the study protocol.

Body mass index (BMI) was calculated as weight $(\mathrm{kg}) /$ [height $(\mathrm{m})]^{2}$. Waist circumference (WC) was defined as the horizontal girth through the center of the umbilical, measured by using a soft ruler at the end of exhalation and before the beginning of inspiration. Patients who kept smoking at the time of admission or had quit smoking for less than 1 year and drank $\geq 12$ times over the past year were considered to have a history of smoking and drinking, respectively. Patients with at least one firstdegree family member having coronary artery disease (CAD) were considered to have a family history of CAD. Patients with hypertension were defined as those with previous definite diagnosis or having systolic/diastolic blood pressure $\geq 140 / 90 \mathrm{mmHg}$ more than two times on different days during the baseline hospitalization. Patients with T2DM were defined as those with previous 


\section{Patients admitted for nonemergent coronary procedures between June 1st, 2018 and June 1st, $2019(n=4187)$}

Patients meeting inclusion criteria $(n=2579)$ :

- age $\geq 18$ years

- diagnosed with NSTE-ACS (including NSTEMI and UA)

- successfully underwent $\mathrm{PCl}$ (defined as residual stenosis of the target lesion $<30 \%$ by visual examination or quantitative assessment, and the absence of serious adverse cardiac events during hospitalization)

Patients meeting exclusion criteria $(n=317)$ :

- missing baseline data

- previous history of coronary artery bypass grafting

- suspected familial hypertriglyceridemia: fasting $T G \geq 5.65 \mathrm{mmol} / \mathrm{L}$ or $500 \mathrm{mg} / \mathrm{dL}$

- extreme elevation of $\mathrm{BMI}: \mathrm{BMI}>45 \mathrm{~kg} / \mathrm{m}^{2}$

- taking TG-lowering medications before admission

- definite or plausible type 1 diabetes mellitus

- acute or chronic infectious diseases

- active bleeding (except for menstruation) or definite/suspected hemorrhagic diseases

- situations requiring long-term oral anticoagulants

- cardiogenic shock, severe heart failure or receiving mechanical circulatory support device

- severe hepatic dysfunction or active hepatic diseases

- severe renal dysfunction with eGFR $<30 \mathrm{~mL} / \mathrm{min} / 1.73 \mathrm{~m}^{2}$ or renal replacement therapy

- malignant tumor or life expectancy of less than 1 year

- unwilling or unable to complete the follow-up procedure after discharge

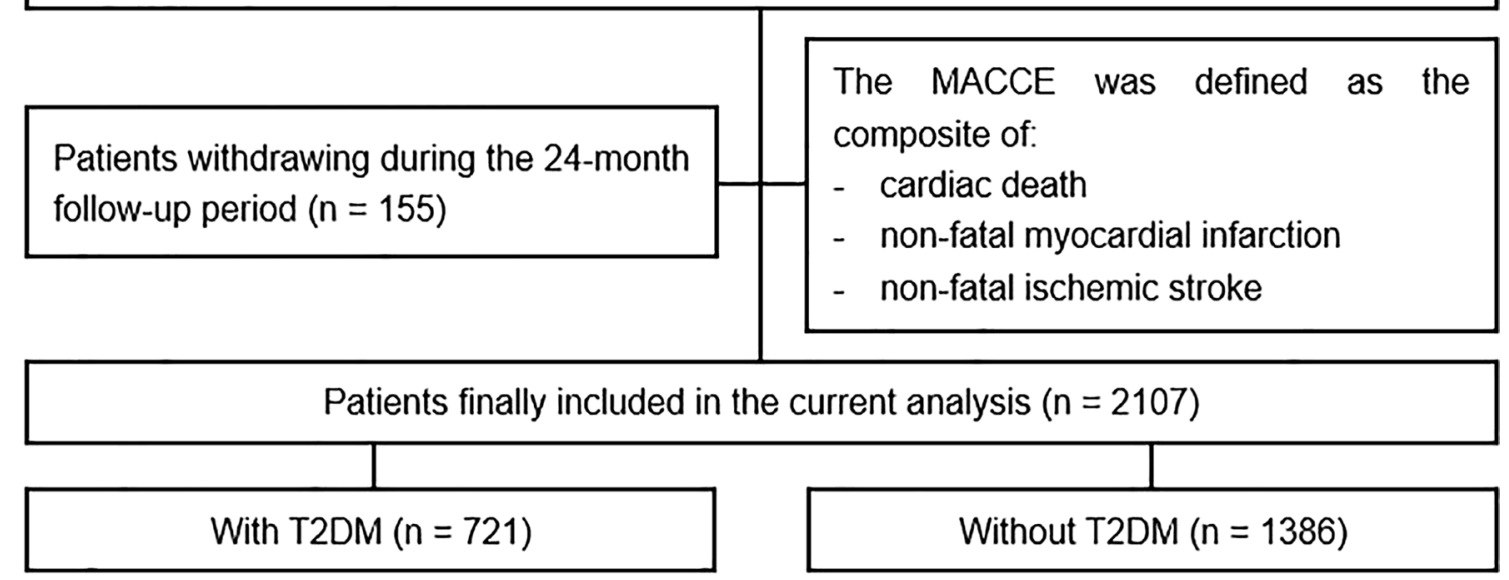

Fig. 1 Flow diagram for the enrollment of study population. NSTE-ACS non-ST-segment elevation acute coronary syndrome, NSTEMI non-ST-segment elevation myocardial infarction, UA unstable angina, $P C l$ percutaneous coronary intervention, $T G$ triglyceride, $B M I$ body mass index, eGFR estimated glomerular filtration rate, MACCE major adverse cardiac and cerebrovascular events, T2DM type 2 diabetes mellitus 
definite diagnosis or newly confirmed T2DM based on the practical guidelines [28]. Previous medical history of myocardial infarction (MI), PCI, stroke, and peripheral artery disease (PAD) was obtained from self-reported information and then confirmed by relevant medical records. Stroke included cerebral infarction and transient ischemic attack. PAD was defined as the artery disease that happened other than the aorta and coronary arteries with stenosis $\geq 50 \%$ and associated ischemic symptoms and/or signs.

Laboratory indices, including lipid profiles [triglyceride (TG), total cholesterol (TC), low-density lipoprotein cholesterol (LDL-C), and high-density lipoprotein cholesterol (HDL-C)], high-sensitivity C-reactive protein (hs-CRP), Creatinine, estimated glomerular filtration rate (eGFR), uric acid, and glycemic parameters [fasting blood glucose (FBG) and glycosylated hemoglobin A1c (HbA1c)], were examined with standard techniques at the core laboratory by using peripheral venous blood samples extracted in the case of fasting $\geq 8 \mathrm{~h}$ before coronary procedures. Left ventricular ejection fraction (LVEF) was estimated by echocardiography with the modified Simpson rule.

Medications including angiotensin-converting enzyme inhibitor (ACEI)/angiotensin receptor blocker (ARB), antiplatelet therapy, $\beta$-blocker, statins, oral antidiabetic agents, and insulin were all prescribed referring to the recommendations of practice guidelines [27] and at the discretion of the chief physicians who were unaware of the study protocol.

Coronary procedures including coronary angiography and PCI were performed by interventional cardiologists who were blind to the study protocol, in line with present guidelines in China [29]. Coronary procedural information was interpreted and recorded by two independent and experienced cardiologists who were unaware of the study protocol. Conflicts confronted during the interpretation of coronary procedures were resolved by turning to a third experienced cardiologist. Coronary lesion characteristics were described in compliance with corresponding guidelines [30]. The synergy between PCI with taxus and cardiac surgery (SYNTAX) score was determined by an online calculator (www.syntaxscore.com) to evaluate the coronary lesion complexity. Complete revascularization was defined as successful interventional procedures (residual stenosis $\leq 30 \%$ ) in all coronary lesions with reference diameter $\geq 1.5 \mathrm{~mm}$ and stenosis $\geq 50 \%$.

\section{Calculation of IR surrogates}

The formulas for calculation of various IR surrogates and the cut-off value for identifying IR in previous studies were listed as follows:

\begin{tabular}{|c|c|c|c|}
\hline $\begin{array}{l}\text { IR } \\
\text { surrogates }\end{array}$ & Formulas & $\begin{array}{l}\text { Cut-off } \\
\text { value }\end{array}$ & References \\
\hline TyG index & $\begin{array}{l}\text { Ln [fasting TG }(\mathrm{mg} / \mathrm{dL}) \times \mathrm{FBG} \\
(\mathrm{mg} / \mathrm{dL}) / 2]\end{array}$ & 4.68 & [10] \\
\hline VAl & $\begin{array}{l}\text { Male: }[\mathrm{WC}(\mathrm{cm}) / \\
(39.68+1.88 \times \mathrm{BMI})] \times[\text { fast- } \\
\text { ing TG } \\
(\mathrm{mmol} / \mathrm{L}) / 1.03] \times[1.31 / \text { fast- } \\
\text { ing HDL-C }(\mathrm{mmol} / \mathrm{L})] \\
\text { Female: }[\mathrm{WC}(\mathrm{cm}) / \\
(36.58+1.89 \times \mathrm{BMI})] \times[\text { fast- } \\
\text { ing TG } \\
(\mathrm{mmol} / \mathrm{L}) / 0.81] \times[1.52 / \text { fast- } \\
\text { ing HDL-C }(\mathrm{mmol} / \mathrm{L})]\end{array}$ & 1.65 & {$[11,31]$} \\
\hline CVAI & $\begin{array}{l}\text { Male: }-267.93+0.68 \times \text { ag } \\
\text { e }+0.03 \times \mathrm{BMI}+4.00 \times \mathrm{WC} \\
(\mathrm{cm})+22.00 \times \mathrm{Lg}[\text { fasting TG } \\
(\mathrm{mmol} / \mathrm{L})]-16.32 \times \text { fasting } \\
\mathrm{HDL}-\mathrm{C}(\mathrm{mmol} / \mathrm{L}) \\
\text { Female: }-187.32+1.71 \times \mathrm{a} \\
\text { ge }+4.32 \times \mathrm{BMl}+1.12 \times \mathrm{WC} \\
(\mathrm{cm})+39.76 \times \mathrm{Lg}[\text { fasting TG } \\
(\mathrm{mmol} / \mathrm{L})]-11.66 \times \text { fasting } \\
\mathrm{HDL}-\mathrm{C}(\mathrm{mmol} / \mathrm{L})\end{array}$ & $\begin{array}{l}\text { Not appli- } \\
\text { cable }\end{array}$ & [12] \\
\hline LAP & $\begin{array}{l}\text { Male: }[W C(\mathrm{~cm})-65] \times[\text { fast- } \\
\text { ing TG }(\mathrm{mmol} / \mathrm{L})] \\
\text { Female: }[W C \\
(\mathrm{cm})-58] \times[\text { fasting TG } \\
(\mathrm{mmol} / \mathrm{L})]\end{array}$ & 42.5 & {$[13,31]$} \\
\hline $\mathrm{TG} / \mathrm{HDL}-\mathrm{C}$ & $\begin{array}{l}\text { Fasting TG }(\mathrm{mg} / \mathrm{dL}) \text { / fasting } \\
\mathrm{HDL}-\mathrm{C}(\mathrm{mg} / \mathrm{dL})\end{array}$ & 3.50 & [14] \\
\hline
\end{tabular}

\section{Follow-up and endpoint}

Patients who met the inclusion and exclusion criteria were then routinely followed up every 3 months after discharge, via telephone or outpatient service. All patients were followed up for 24 months unless withdrawal or death occurred. The observational endpoint of the present study was the major adverse cardiovascular and cerebrovascular events (MACCE), which was defined as the composite of cardiac death, non-fatal MI, and non-fatal ischemic stroke. All events were documented and further verified by referring to relevant medical records if indistinct information was acquired. The MACCE was considered to be the first adverse event that occurred during each patient's follow-up.

\section{Statistical analysis}

Continuous variates were described as mean with standard deviation or median with interquartile range, and the comparison between two groups was examined by T-test or Mann-Whitney U-test correspondingly. Nominal variates were described as number with percentage and the comparison between two groups was examined by Chisquare test (with or without continuity correction) or Fisher's exact test accordingly. 
Cumulative no MACCE survival rates according to the median of each IR surrogate were evaluated by Kaplan-Meier analysis, and the differences between higher and lower median groups were detected by logrank test. Unadjusted and fully adjusted Cox regression analyses were performed to evaluate the value of each IR surrogate on the prediction of MACCE. The model used in fully adjusted Cox regression analysis included smoking history, hypertension, T2DM, previous MI, previous PCI, previous stroke, clinical diagnosis, TC, hs-CRP, eGFR, HbA1c, LVEF, ACEI/ ARB at discharge, oral antidiabetic agents at discharge, insulin at discharge, left main artery (LM) disease, three-vessel disease, chronic total occlusion, SYNTAX score, complete revascularization, and number of stents. The variates were selected based on univariate analysis $(\mathrm{P}<0.05)$ (Additional file 1: Table S1) and clinical experience. As determinants of IR surrogates, age, gender, BMI, WC, TG, HDL-C, and FBG were not included. The hazard ratio (HR) and 95\% confidence interval (CI) for MACCE were examined by taking each IR surrogate as a nominal and continuous variate, respectively. When being taken as a nominal variate, the HR was examined by regarding the lower median of each IR surrogate as the reference. When being taken as a continuous variate, each IR surrogate was normalized by the Z-score method to compare the predictive value of them intuitively, then the HR was examined by evaluating 1 normalized unit increase. To identify the effects of medications including statins, oral antidiabetic agents, and insulin on the predictive value of IR surrogates for MACCE, sensitivity analysis was undertaken by stratifying the study population according to whether or not they were taking these medications at admission. Furthermore, the continuous relationship (linear or non-linear) between each IR surrogate and the risk of MACCE was illustrated by restricted cubic spline and examined by the likelihood ratio test.

The diagnostic value of each IR surrogate for MACCE was assessed by receiver operating characteristic (ROC) analysis. The area under the ROC curves (AUCs) were determined and then compared by the Z-test. Moreover, Harrell's C-index, continuous net reclassification improvement (NRI), and integrated discrimination improvement (IDI) were determined to evaluate the incremental effect of each IR surrogate on risk stratification.

Similar statistical analyses described above were performed in subgroups with and without T2DM, respectively. Data analyses were performed with IBM SPSS Statistics (version 26.0), the $\mathrm{R}$ Programming Language (version 3.6.3), and MedCalc (version 19.1). P-value (twotailed) $<0.05$ suggested statistical significance.

\section{Results}

Overall, 2107 patients $(60.02 \pm 9.03$ years, $28.0 \%$ female) who met the enrollment criteria and completed the follow-up were ultimately brought into the present study. During the 24-month follow-up, 18 (0.9\%) cardiac deaths, 124 (5.9\%) non-fatal MIs, and 46 (2.2\%) non-fatal ischemic strokes were recorded. One of the patients experienced non-fatal MI followed by nonfatal ischemic stroke. Thus, a total of 187 (8.9\%) MACCEs were finally taken into the present analysis.

\section{Baseline characteristics of the total population}

The baseline characteristics of the total population were summarized in Table 1 . The levels of IR surrogates including the TyG index, VAI, CVAI, LAP, and TG/ HDL-C were all significantly higher in patients with MACCE. Patients who experienced MACCE showed higher proportions of female, T2DM, hypertension, previous MI, previous PCI, and previous stroke, higher levels of WC, TG, TC, FBG, and HbA1c, while lower levels of HDL-C and LVEF. Meanwhile, more patients were diagnosed with NSTEMI and treated with ACEI/ $A R B$, oral antidiabetic agents, and insulin in the MACCE group. As for coronary procedural information, patients in the MACCE group exhibited more LM disease, three-vessel disease, and chronic total occlusion, less complete revascularization, and higher SYNTAX score.

\section{Predictive value of IR surrogates for the risk of MACCE}

The incidence of MACCE in patients with higher median of the TyG index (14.4\% vs. 3.3\%), VAI ( $12.7 \%$ vs. $5.0 \%)$, CVAI $(11.9 \%$ vs. $5.9 \%)$, LAP $(12.2 \%$ vs. $5.6 \%)$, and TG/HDL-C (12.0\% vs. $5.8 \%)$ was significantly higher than that in those with lower median (all $\mathrm{P}<0.001$ ) (Fig. 2). Meanwhile, the Kaplan-Meier analysis revealed that the patients with higher median of each IR surrogate showed significantly lower no MACCE survival rates than those with lower median (all log-rank $\mathrm{P}<0.001$ ) (Fig. 3).

The results of unadjusted and fully adjusted Cox regression analyses showed that the TyG index, compared with other IR surrogates, was exhibited to be the strongest risk predictor for MACCE with the highest HR, despite taking the lower median as reference (unadjusted HR 4.660, 95\% CI 3.227-6.729, P <0.001; adjusted HR 3.805, 95\% CI 2.581-5.608, $\mathrm{P}<0.001$ ) or examining 1 normalized unit increase (unadjusted HR 2.049, 95\% CI 1.793-2.343, $\mathrm{P}<0.001$; adjusted HR 1.847, 95\% CI $1.564-2.181, \mathrm{P}<0.001$ ). The predictive value of other IR surrogates was significant but relatively weaker than that of the TyG index (Table 2). 
Table 1 Baseline characteristics of the total population

\begin{tabular}{|c|c|c|c|c|}
\hline & $\begin{array}{l}\text { Total population } \\
(\mathrm{n}=2107)\end{array}$ & $\begin{array}{l}\text { MACCE } \\
(n=187)\end{array}$ & $\begin{array}{l}\text { Non-MACCE } \\
(n=1920)\end{array}$ & P-value \\
\hline \multicolumn{5}{|l|}{ IR surrogates } \\
\hline TyG index & $8.87 \pm 0.60$ & $9.30 \pm 0.62$ & $8.82 \pm 0.58$ & $<0.001$ \\
\hline $\mathrm{VAl}$ & $2.84 \pm 1.98$ & $3.95 \pm 2.54$ & $2.73 \pm 1.88$ & $<0.001$ \\
\hline CVAl & $132.18 \pm 45.07$ & $151.37 \pm 45.35$ & $130.31 \pm 44.61$ & $<0.001$ \\
\hline LAP & $51.04 \pm 40.28$ & $76.31 \pm 55.99$ & $48.58 \pm 37.53$ & $<0.001$ \\
\hline TG/HDL-C & $4.35 \pm 2.86$ & $5.74 \pm 3.51$ & $4.21 \pm 2.75$ & $<0.001$ \\
\hline Age, years & $60.02 \pm 9.03$ & $61.25 \pm 9.89$ & $59.90 \pm 8.93$ & 0.073 \\
\hline Gender, female, n (\%) & $591(28.0)$ & $65(34.8)$ & $526(27.4)$ & 0.032 \\
\hline $\mathrm{BMI}, \mathrm{kg} / \mathrm{m}^{2}$ & $26.08 \pm 3.21$ & $26.43 \pm 3.30$ & $26.05 \pm 3.20$ & 0.115 \\
\hline$W C, c m$ & $91.39 \pm 12.39$ & $95.24 \pm 13.06$ & $91.01 \pm 12.26$ & $<0.001$ \\
\hline Heart rate, bpm & $69.80 \pm 10.15$ & $71.03 \pm 10.40$ & $69.68 \pm 10.12$ & 0.083 \\
\hline SBP, mmHg & $130.17 \pm 16.47$ & $129.79 \pm 17.95$ & $130.21 \pm 16.33$ & 0.741 \\
\hline $\mathrm{DBP}, \mathrm{mmHg}$ & $76.94 \pm 9.78$ & $76.00 \pm 10.26$ & $77.03 \pm 9.73$ & 0.166 \\
\hline Smoking history, n (\%) & $1195(56.7)$ & $103(55.1)$ & $1092(56.9)$ & 0.636 \\
\hline Drinking history, n (\%) & $495(23.5)$ & $44(23.5)$ & $451(23.5)$ & 0.990 \\
\hline Family history of CAD, n (\%) & $218(10.3)$ & $14(7.5)$ & $204(10.6)$ & 0.179 \\
\hline \multicolumn{5}{|l|}{ Medical history, n (\%) } \\
\hline T2DM & $721(34.2)$ & $89(47.6)$ & $632(32.9)$ & $<0.001$ \\
\hline Hypertension & 1305 (61.9) & $129(69.0)$ & $1176(61.3)$ & 0.038 \\
\hline Previous MI & $440(20.9)$ & $67(35.8)$ & $373(19.4)$ & $<0.001$ \\
\hline Previous $\mathrm{PCl}$ & $359(17.0)$ & $45(24.1)$ & $314(16.4)$ & 0.007 \\
\hline Previous stroke & $235(11.2)$ & $31(16.6)$ & $204(10.6)$ & 0.014 \\
\hline Previous PAD & $73(3.5)$ & $6(3.2)$ & $67(3.5)$ & 0.841 \\
\hline Clinical diagnosis, n (\%) & & & & 0.021 \\
\hline NSTEMI & $1750(83.1)$ & $43(23.0)$ & $314(16.4)$ & \\
\hline UA & $357(16.9)$ & $144(77.0)$ & $1606(83.6)$ & \\
\hline \multicolumn{5}{|l|}{ Laboratory tests } \\
\hline $\mathrm{TG}, \mathrm{mmol} / \mathrm{L}$ & $1.71 \pm 0.90$ & $2.21 \pm 1.07$ & $1.66 \pm 0.87$ & $<0.001$ \\
\hline $\mathrm{TC}, \mathrm{mmol} / \mathrm{L}$ & $4.17 \pm 1.04$ & $4.38 \pm 1.11$ & $4.14 \pm 1.03$ & 0.003 \\
\hline $\mathrm{LDL}-\mathrm{C}, \mathrm{mmol} / \mathrm{L}$ & $2.52 \pm 0.88$ & $2.60 \pm 0.90$ & $2.51 \pm 0.88$ & 0.208 \\
\hline $\mathrm{HDL}-\mathrm{C}, \mathrm{mmol} / \mathrm{L}$ & $0.99 \pm 0.23$ & $0.95 \pm 0.22$ & $0.99 \pm 0.23$ & 0.029 \\
\hline hs-CRP, mg/L & $1.27(0.57,3.26)$ & $1.46(0.66,3.95)$ & $1.25(0.55,3.18)$ & 0.084 \\
\hline Creatinine, $\mu \mathrm{mol} / \mathrm{L}$ & $75.86 \pm 16.61$ & $75.30 \pm 15.85$ & $75.91 \pm 16.69$ & 0.630 \\
\hline $\mathrm{eGFR}, \mathrm{mL} / \mathrm{min} / 1.73 \mathrm{~m}^{2}$ & $93.62 \pm 19.95$ & $92.22 \pm 20.40$ & $93.75 \pm 19.91$ & 0.317 \\
\hline Uric acid, $\mu \mathrm{mol} / \mathrm{L}$ & $344.87 \pm 80.82$ & $351.16 \pm 82.23$ & $344.26 \pm 80.67$ & 0.265 \\
\hline $\mathrm{FBG}, \mathrm{mmol} / \mathrm{L}$ & $6.11 \pm 1.88$ & $7.33 \pm 3.08$ & $5.99 \pm 1.67$ & $<0.001$ \\
\hline $\mathrm{HbA1c} \%$ & $6.26 \pm 1.18$ & $6.90 \pm 1.49$ & $6.19 \pm 1.13$ & $<0.001$ \\
\hline LVEF, \% & $64.02 \pm 6.69$ & $62.53 \pm 8.04$ & $64.16 \pm 6.53$ & 0.008 \\
\hline \multicolumn{5}{|l|}{ Medications at admission, n (\%) } \\
\hline ACEI/ARB & $470(22.3)$ & $46(24.6)$ & $424(22.1)$ & 0.430 \\
\hline DAPT & $632(30.0)$ & $58(31.0)$ & $574(29.9)$ & 0.750 \\
\hline Aspirin & $1105(52.4)$ & $103(55.1)$ & $1002(52.2)$ & 0.450 \\
\hline P2Y12 inhibitors & $672(31.9)$ & $64(34.2)$ & $608(31.7)$ & 0.474 \\
\hline$\beta$-blocker & $468(22.2)$ & $51(27.3)$ & $417(21.7)$ & 0.081 \\
\hline Statins & $649(30.8)$ & $64(34.2)$ & $585(30.5)$ & 0.288 \\
\hline Oral antidiabetic agents & $375(17.8)$ & $48(25.7)$ & $327(17.0)$ & 0.003 \\
\hline Insulin & $198(9.4)$ & $29(15.5)$ & $169(8.8)$ & 0.003 \\
\hline \multicolumn{5}{|l|}{ Medications at discharge, $\mathrm{n}(\%)$} \\
\hline ACEI/ARB & $1455(69.1)$ & $154(82.4)$ & $1301(67.8)$ & $<0.001$ \\
\hline
\end{tabular}


Table 1 (continued)

\begin{tabular}{|c|c|c|c|c|}
\hline & $\begin{array}{l}\text { Total population } \\
(n=2107)\end{array}$ & $\begin{array}{l}\text { MACCE } \\
(n=187)\end{array}$ & $\begin{array}{l}\text { Non-MACCE } \\
(n=1920)\end{array}$ & P-value \\
\hline DAPT & $2106(100.0)$ & $187(100.0)$ & $1919(99.9)$ & $>0.999$ \\
\hline Aspirin & $2106(100.0)$ & $187(100.0)$ & 1919 (99.9) & $>0.999$ \\
\hline P2Y12 inhibitors & $2107(100.0)$ & $187(100.0)$ & $1920(100.0)$ & 1.000 \\
\hline$\beta$-blocker & $1910(90.7)$ & $171(91.4)$ & $1739(90.6)$ & 0.696 \\
\hline Statins & 2065 (98.0) & $183(97.9)$ & $1882(98.0)$ & 0.881 \\
\hline Oral antidiabetic agents & $372(17.7)$ & $47(25.1)$ & $325(16.9)$ & 0.005 \\
\hline Insulin & $190(9.0)$ & $27(14.4)$ & $163(8.5)$ & 0.007 \\
\hline \multicolumn{5}{|l|}{ Coronary procedural information } \\
\hline LM disease, $n(\%)$ & $93(4.4)$ & $18(9.6)$ & $75(3.9)$ & $<0.001$ \\
\hline Three-vessel disease, n (\%) & $631(29.9)$ & $77(41.2)$ & $554(28.9)$ & $<0.001$ \\
\hline Chronic total occlusion, n (\%) & $277(13.1)$ & $39(20.9)$ & $238(12.4)$ & 0.001 \\
\hline Diffuse lesion, n (\%) & $508(24.1)$ & $55(29.4)$ & $453(23.6)$ & 0.076 \\
\hline Bifurcation lesion, n (\%) & $435(20.6)$ & $40(21.4)$ & $395(20.6)$ & 0.792 \\
\hline SYNTAX score & $10.62 \pm 5.46$ & $12.84 \pm 6.23$ & $10.40 \pm 5.33$ & $<0.001$ \\
\hline \multicolumn{5}{|l|}{ Target vessel territory, n (\%) } \\
\hline LM & $52(2.5)$ & $8(4.3)$ & $44(2.3)$ & 0.095 \\
\hline LAD & $1379(65.4)$ & $115(61.5)$ & $1264(65.8)$ & 0.234 \\
\hline LCX & $724(34.4)$ & $61(32.6)$ & $663(34.5)$ & 0.599 \\
\hline RCA & $895(42.5)$ & $90(48.1)$ & $805(41.9)$ & 0.101 \\
\hline Complete revascularization, n (\%) & $1237(58.7)$ & $90(48.1)$ & $1147(59.7)$ & 0.002 \\
\hline Number of stents & $1.98 \pm 1.27$ & $2.09 \pm 1.34$ & $1.97 \pm 1.27$ & 0.254 \\
\hline
\end{tabular}

IR insulin resistance, TyG triglyceride-glucose, VAI visceral adiposity index, CVAI Chinese visceral adiposity index, LAP lipid accumulation product, TG/HDL-C triglyceride to high-density lipoprotein cholesterol ratio, BMI body mass index, WC waist circumference, SBP systolic blood pressure, DBP diastolic blood pressure, CAD coronary artery disease, T2DM type 2 diabetes mellitus, MI myocardial infarction, $P C /$ percutaneous coronary intervention, PAD peripheral artery disease, NSTEMI non-ST-segment elevation myocardial infarction, UA unstable angina, TG triglyceride, TC total cholesterol, LDL-C low-density lipoprotein cholesterol, $H D L-C$ highdensity lipoprotein cholesterol, $h \mathrm{~s}$-CRP high-sensitivity C-reactive protein, eGFR estimated glomerular filtration rate, $F B G$ fasting blood glucose, HbA1c glycosylated hemoglobin A1c, LVEF left ventricular ejection fraction, $A C E l$ angiotensin converting enzyme inhibitor, $A R B$ angiotensin receptor blocker, DAPT dual antiplatelet therapy, $L M$ left main artery, SYNTAX synergy between PCI with taxus and cardiac surgery, $L A D$ left anterior descending artery, $L C X$ left circumflex artery, $R C A$ right coronary artery, MACCE major adverse cardiac and cerebrovascular events

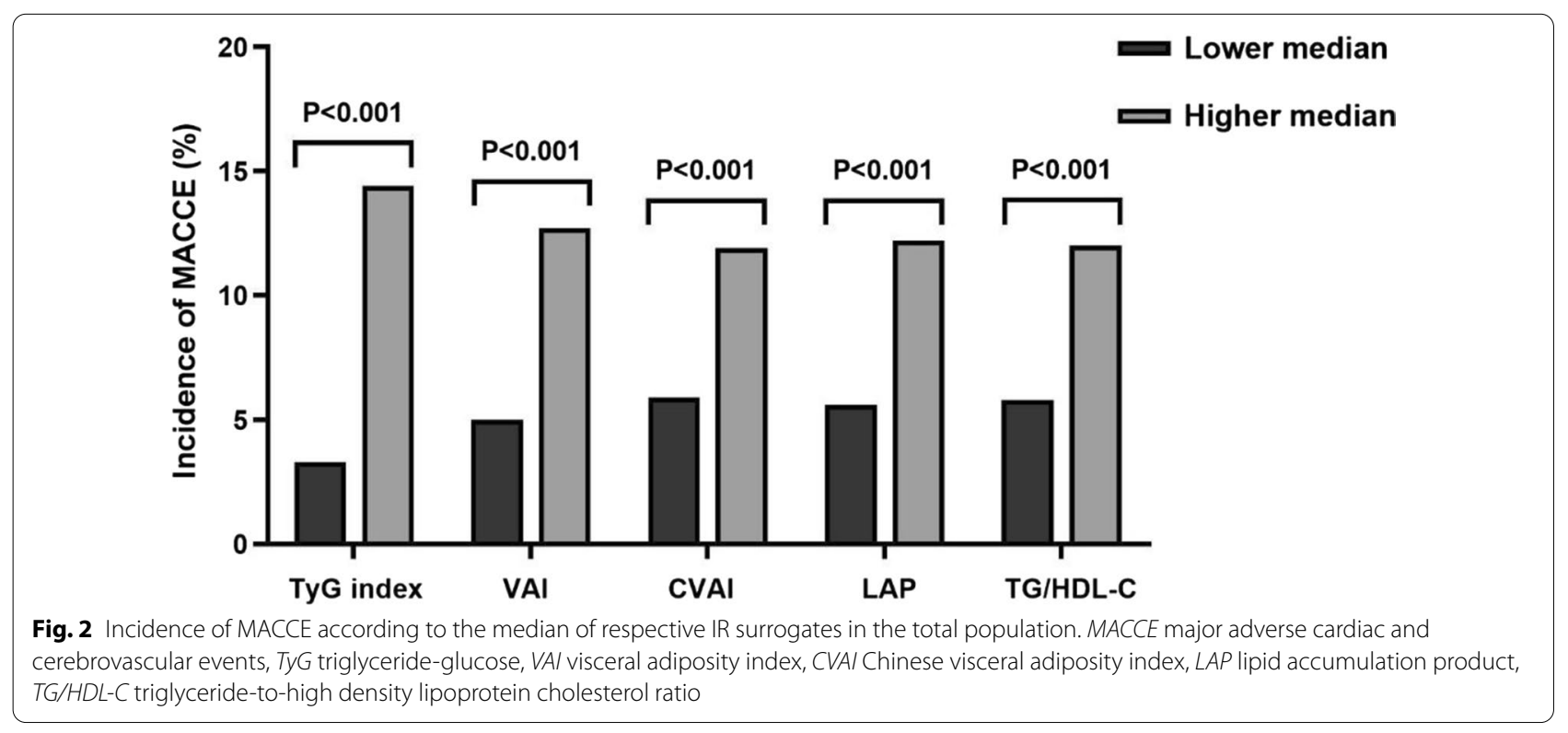




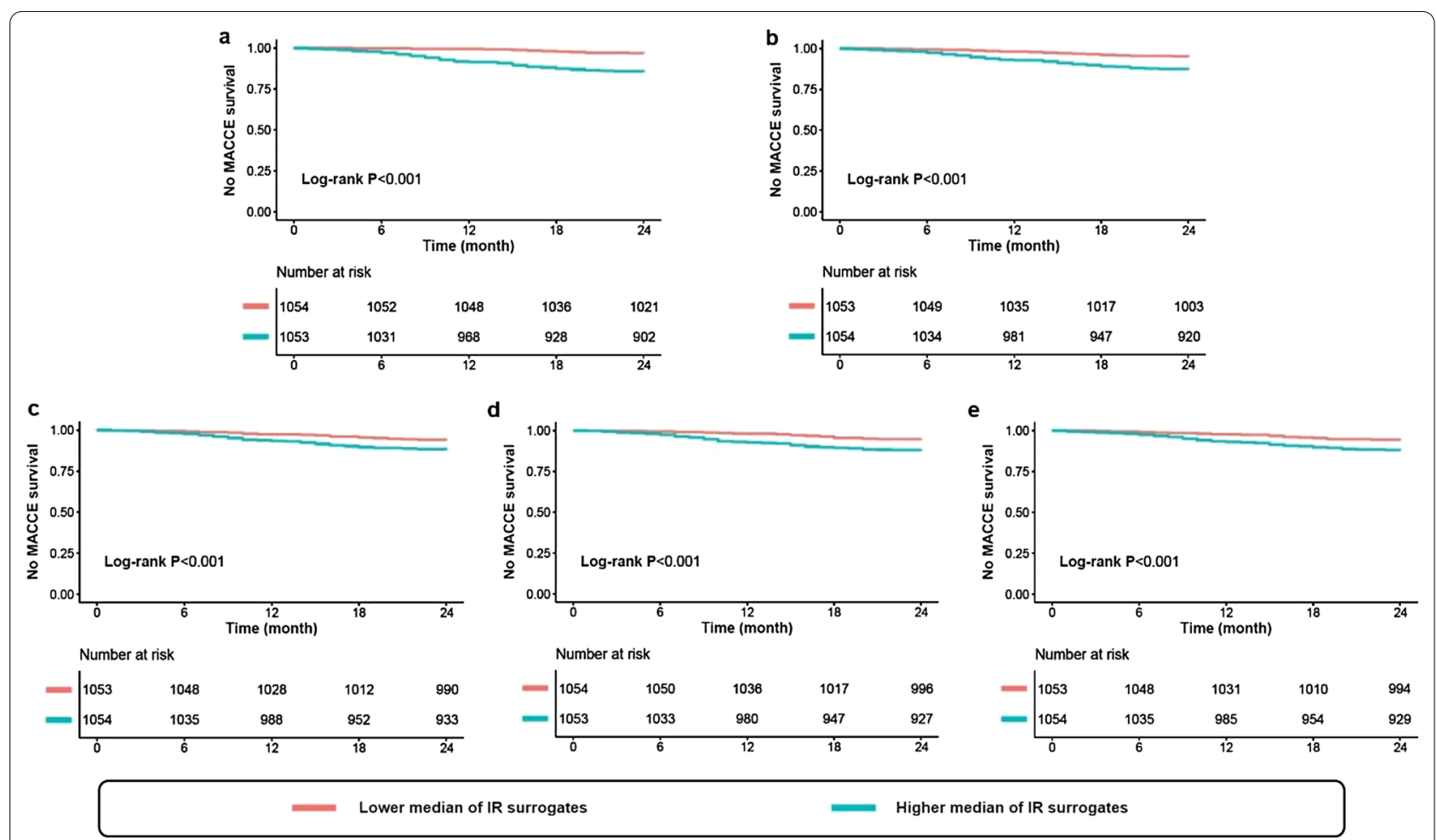

Fig. 3 Kaplan-Meier curves for no MACCE survival according to the median of TyG index (a), VAl (b), CVAI (c), LAP (d), and TG/HDL-C (e) in the total population. MACCE major adverse cardiac and cerebrovascular events, IR insulin resistance

Table 2 Predictive value of various IR surrogates for the risk of MACCE in the total population

\begin{tabular}{|c|c|c|c|c|c|c|}
\hline \multirow[t]{2}{*}{ IR surrogates } & \multirow[t]{2}{*}{ Variate type } & \multirow{2}{*}{$\begin{array}{l}\text { No. MACCE } \\
\text { Lower/Higher }\end{array}$} & \multicolumn{2}{|l|}{ Unadjusted analysis } & \multicolumn{2}{|l|}{ Adjusted analysis $^{c}$} \\
\hline & & & $\mathrm{HR}(95 \% \mathrm{CI})$ & P-value & HR $(95 \% \mathrm{Cl})$ & P-value \\
\hline \multirow[t]{2}{*}{ TyG index } & Nominal $^{\mathrm{a}}$ & $35 / 152$ & $4.660(3.227-6.729)$ & $<0.001$ & $3.805(2.581-5.608)$ & $<0.001$ \\
\hline & Continuous $^{\mathrm{b}}$ & - & $2.049(1.793-2.343)$ & $<0.001$ & $1.847(1.564-2.181)$ & $<0.001$ \\
\hline \multirow[t]{2}{*}{ VAl } & Nominal $^{a}$ & $53 / 134$ & $2.648(1.926-3.639)$ & $<0.001$ & $2.161(1.559-2.996)$ & $<0.001$ \\
\hline & Continuous $^{b}$ & - & $1.517(1.373-1.675)$ & $<0.001$ & $1.420(1.272-1.586)$ & $<0.001$ \\
\hline \multirow[t]{2}{*}{ CVAl } & Nominal $^{a}$ & $62 / 125$ & $2.093(1.544-2.838)$ & $<0.001$ & $1.648(1.203-2.258)$ & 0.002 \\
\hline & Continuous $^{b}$ & - & $1.533(1.339-1.756)$ & $<0.001$ & $1.353(1.171-1.563)$ & $<0.001$ \\
\hline \multirow[t]{2}{*}{ LAP } & Nominal $^{a}$ & $59 / 128$ & $2.273(1.670-3.094)$ & $<0.001$ & $1.764(1.279-2.432)$ & 0.001 \\
\hline & Continuous $^{\mathrm{b}}$ & - & $1.540(1.405-1.689)$ & $<0.001$ & $1.431(1.289-1.589)$ & $<0.001$ \\
\hline \multirow[t]{2}{*}{$\mathrm{TG} / \mathrm{HDL}-\mathrm{C}$} & Nominal $^{\mathrm{a}}$ & $61 / 126$ & $2.146(1.581-2.914)$ & $<0.001$ & $1.757(1.284-2.404)$ & $<0.001$ \\
\hline & Continuous $^{b}$ & - & $1.466(1.319-1.629)$ & $<0.001$ & $1.395(1.241-1.569)$ & $<0.001$ \\
\hline
\end{tabular}

IR insulin resistance, TyG triglyceride-glucose, VAI visceral adiposity index, CVAI Chinese visceral adiposity index, LAP lipid accumulation product, TG/HDL-C triglyceride-to-high density lipoprotein cholesterol ratio, MACCE major adverse cardiac and cerebrovascular events, $H R$ hazard ratio, $C l$ confidence interval ${ }^{a}$ The HR was examined by regarding the lower median as reference

${ }^{\mathrm{b}}$ The HR was examined by evaluating 1 normalized unit increase

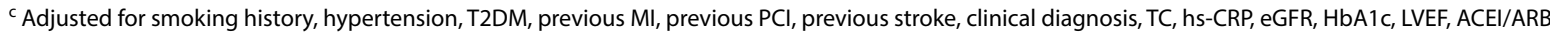
at discharge, oral antidiabetic agents at discharge, insulin at discharge, LM disease, three-vessel disease, chronic total occlusion, SYNTAX score, complete revascularization, and number of stents

Since the determinants of IR surrogates may be affected by medications, especially lipid-lowering and hypoglycemic agents, sensitivity analysis was performed and revealed that each IR surrogate was robustly associated with the risk of MACCE, regardless of whether a statin, oral antidiabetic agent, or 


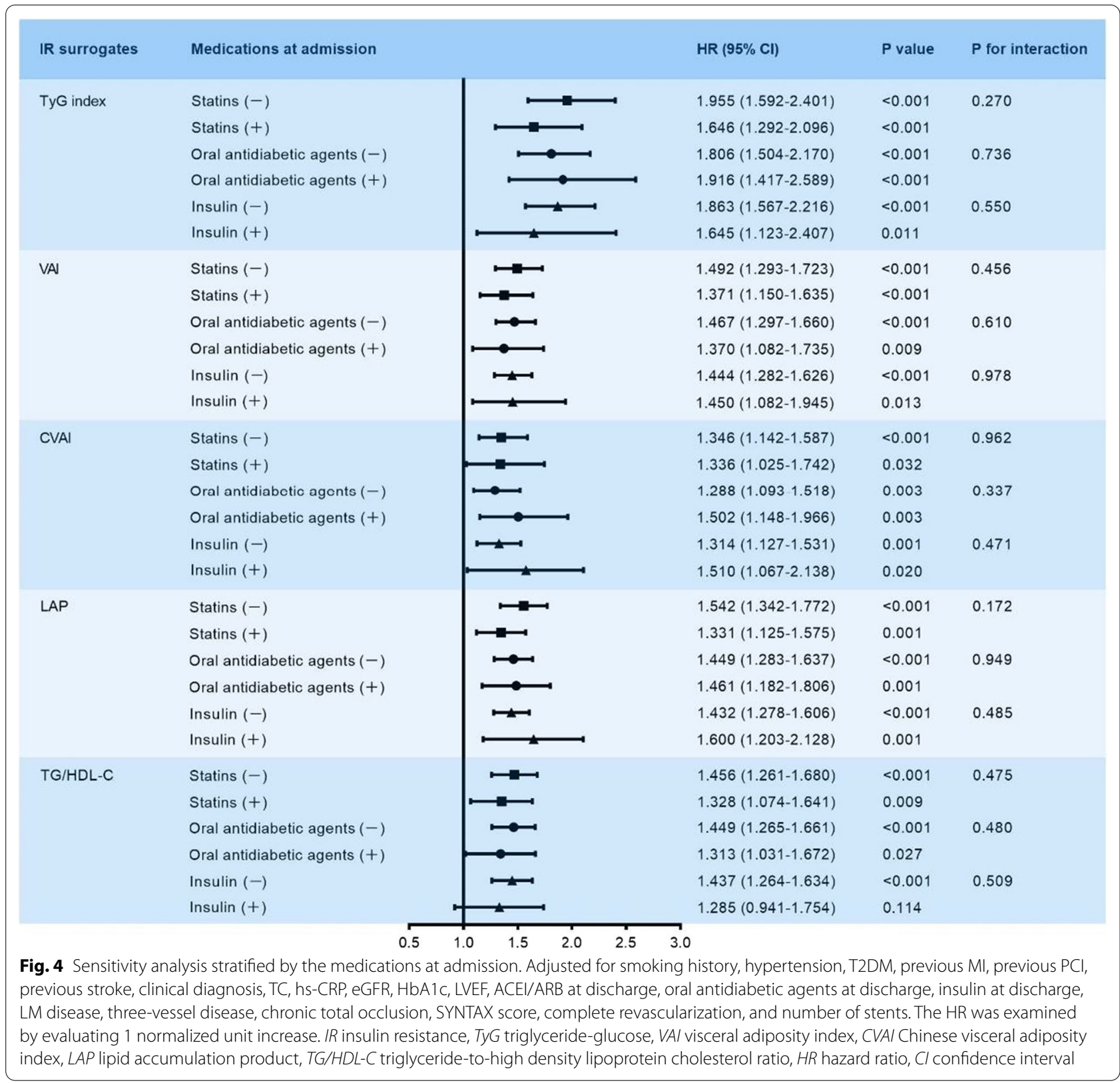

insulin was administered at admission (all $\mathrm{P}$ for interaction >0.05) (Fig. 4). Moreover, restricted cubic spline analysis elucidated that there was a linear association between each IR surrogate and the risk of MACCE (all $\mathrm{P}$ for non-linear association $<0.001$ ) (Fig. 5).

\section{Diagnostic performance of IR surrogates for MACCE}

The diagnostic performance of each IR surrogate for MACCE was assessed and compared by ROC curve analysis. The TyG index $(0.715,95 \%$ CI $0.695-0.734)$ displayed the highest diagnostic ability manifested as the maximum AUC, in comparison with VAI $(0.673,95 \%$ CI
$0.652-0.693$, $\mathrm{P}$ for comparison $=0.001)$, CVAI $(0.628$, 95\% CI $0.607-0.649$, P for comparison $<0.001)$, LAP $(0.670,95 \%$ CI $0.650-0.691$, $\mathrm{P}$ for comparison $=0.001)$, and TG/HDL-C $(0.651,95 \%$ CI $0.630-0.671$, P for comparison $<0.001$ ) (Table 3; Fig. 6). In addition, the cut-off value, sensitivity, and specificity for each IR surrogate were calculated, respectively (details shown in Table 3).

\section{Incremental effect of IR surrogates on risk stratification}

The addition of the TyG index, in comparison with other IR surrogates, exhibited the maximum enhancement on risk stratification for MACCE on the basis of the baseline 


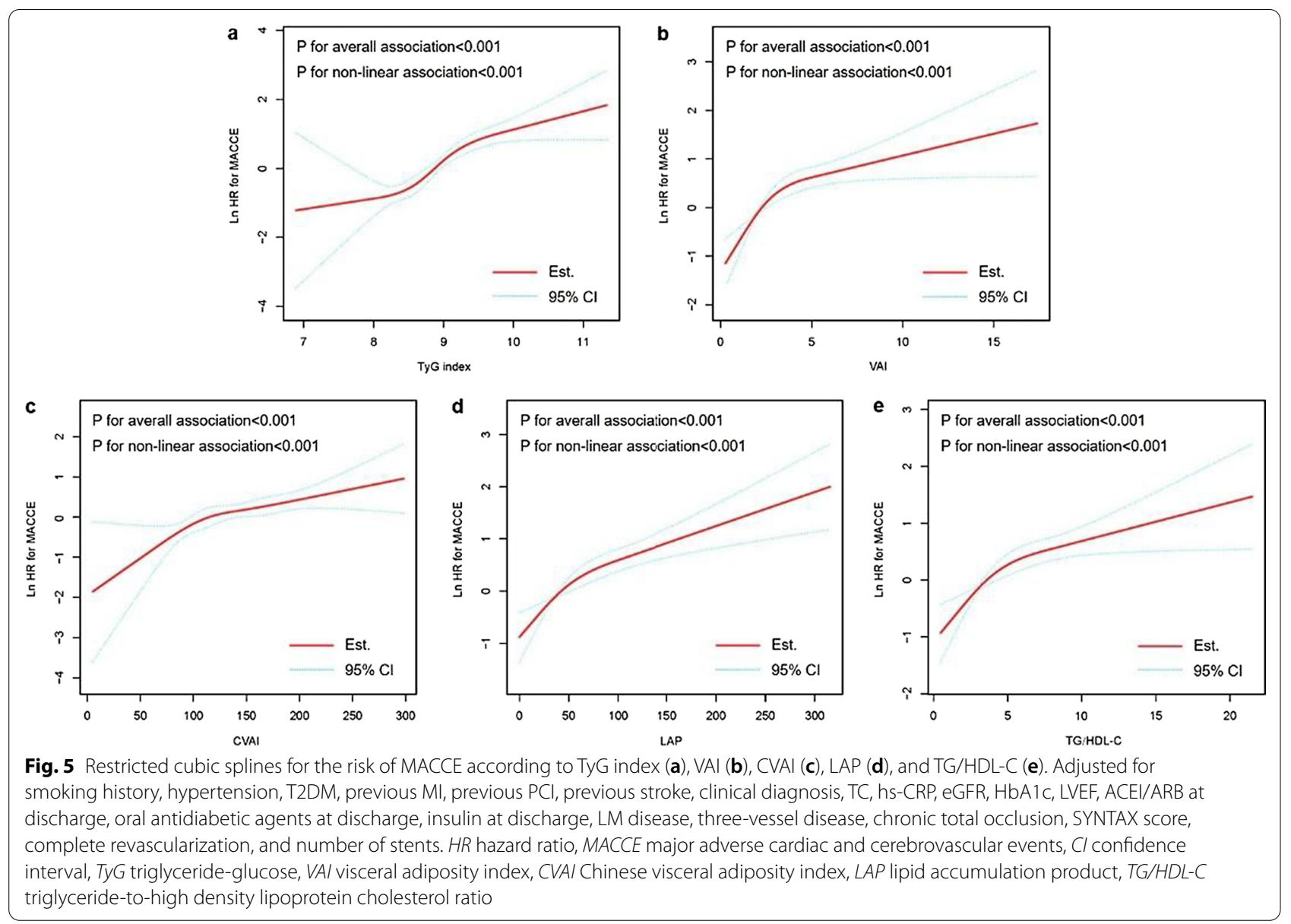

Table 3 Diagnostic performance of IR surrogates for MACCE in the total population

\begin{tabular}{lllcccc}
\hline & \multicolumn{2}{l}{ AUC } & & Cut-off value & Sensitivity, \% & Specificity, \% \\
\cline { 2 - 4 } & Est. $(\mathbf{9 5 \%}$ Cl) & P-value & P for comparison & & & \\
\hline TyG index & $0.715(0.695-0.734)$ & $<0.001$ & - & 8.85 & 81.28 & 53.13 \\
VAl & $0.673(0.652-0.693)$ & $<0.001$ & 0.001 & 2.20 & 75.40 & 49.84 \\
CVAI & $0.628(0.607-0.649)$ & $<0.001$ & $<0.001$ & 117.38 & 80.21 & 40.47 \\
LAP & $0.670(0.650-0.691)$ & $<0.001$ & 0.001 & 56.94 & 54.55 & 70.68 \\
TG/HDL-C & $0.651(0.630-0.671)$ & $<0.001$ & $<0.001$ & 3.02 & 81.82 & 40.94 \\
\hline
\end{tabular}

TyG triglyceride-glucose, VAI visceral adiposity index, CVAI Chinese visceral adiposity index, LAP lipid accumulation product, TG/HDL-C triglyceride-to-high density lipoprotein cholesterol ratio, $A U C$ area under the ROC curve, $\mathrm{Cl}$ confidence interval

model including recognized risk factors (smoking history, hypertension, T2DM, previous MI, previous PCI, previous stroke, TC, eGFR, HbA1c, LVEF, LM disease, threevessel disease, SYNTAX score, and number of stents), in terms of increased Harrell's C-index $(0.708,95 \%$ CI 0.672-0.744 for baseline model vs. 0.758 , 95\% CI $0.726-$ 0.791 for baseline model + TyG index, $\mathrm{P}<0.001$ ), and significant continuous NRI $(0.255,95 \%$ CI $0.145-0.320$, $\mathrm{P}<0.001)$ and IDI $(0.033,95 \%$ CI $0.012-0.058, \mathrm{P}<0.001)$.
Significant but relatively minor incremental effects were obtained after adding other IR surrogates into the baseline model (Table 4).

\section{Subgroup analysis based on T2DM}

The total population was divided into two subgroups (with T2DM, $\mathrm{n}=721$ and without T2DM, $\mathrm{n}=1386$ ). The incidence of MACCE in subgroups with and without T2DM was $12.3 \%$ and $7.1 \%$, respectively. The differences 


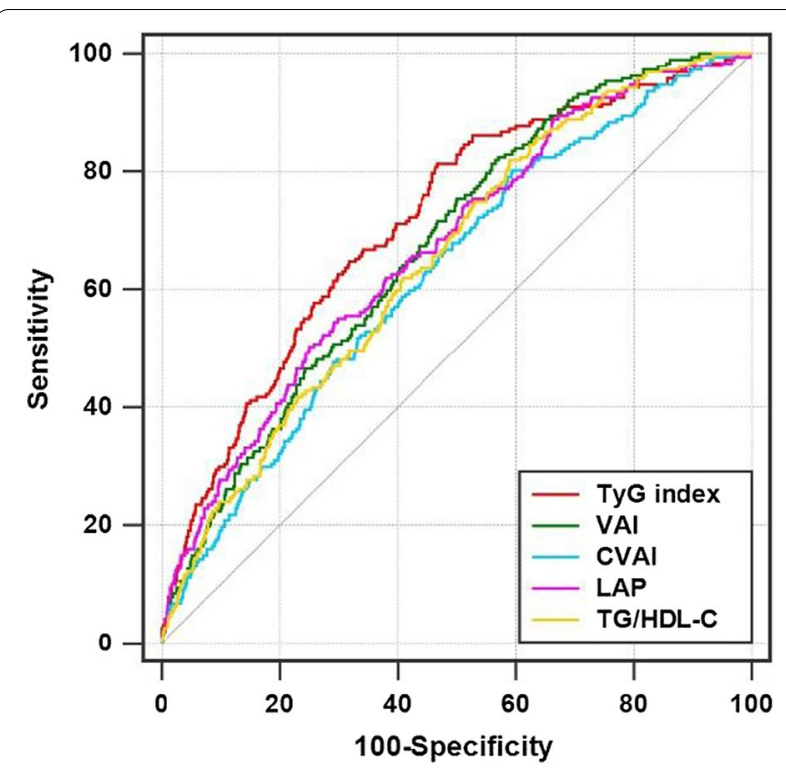

Fig. 6 ROC curves evaluating the diagnostic performance of each IR surrogate for MACCE in the total population. TyG triglyceride-glucose, VAl visceral adiposity index, CVAl Chinese visceral adiposity index, LAP lipid accumulation product, TG/HDL-C triglyceride-to-high density lipoprotein cholesterol ratio

in WC, previous MI, TG, FBG, HbA1c, ACEI/ARB, LM disease, and SYNTAX score between MACCE and nonMACCE group remained significant in subgroups with and without T2DM. However, the discrepancies in gender, previous PCI, TC, three-vessel disease, and chronic total occlusion were only significant in the subgroup with T2DM, while the differences in age, previous stroke, LVEF, $\beta$-blocker, complete revascularization were only significant in the subgroup without T2DM (Additional file 1: Table S2).

In both subgroups, the incidence of MACCE in patients with higher median of each IR surrogate was significantly higher than that in those with lower median (Additional file 2: Figure S1). Similar to the KaplanMeier analysis in the total population, the differences in no MACCE survival rates between higher and lower median of each IR surrogate remained significant in subgroups with and without T2DM (Additional file 2: Figure S2). In unadjusted and fully adjusted Cox regression analyses, the TyG index was consistently shown to have the most powerful risk prediction ability in both subgroups, despite being taken as a nominal and continuous variate. For other IR surrogates, significant but relatively weaker predictive abilities were obtained (Additional file 1: Table S3).

As for ROC curve analysis, the TyG index exhibited the highest AUC among all IR surrogates in subgroups with and without T2DM. Of note, the difference in AUCs was not significant between the TyG index and LAP in the subgroup with T2DM (Additional file 1: Table S4; Additional file 2: Figure S3). Moreover, the TyG index continued to exhibit the strongest incremental effect on risk stratification beyond the baseline model in both subgroups. Nevertheless, minor or insignificant incremental effects were acquired with the addition of other IR surrogates into the baseline model (Additional file 1: Table S5).

\section{Discussion}

The current analyses showed that compared with other IR surrogates, the TyG index consistently showed the most powerful ability on risk prediction, despite the adjustment of confounding factors. The TyG index exhibited the strongest diagnostic value for MACCE with the highest AUC. After being introduced into the baseline model, the TyG index, in comparison with other IR surrogates, exhibited the maximum incremental effect on risk stratification for MACCE. For other IR surrogates, significant but relatively weaker abilities on risk prediction and stratification were obtained. The results were

Table 4 Incremental ability of various IR surrogates on the prediction of MACCE in the total population

\begin{tabular}{|c|c|c|c|c|c|c|c|}
\hline & \multicolumn{3}{|l|}{ Harrell's C-index } & \multicolumn{2}{|l|}{ Continuous NRI } & \multicolumn{2}{|l|}{ IDI } \\
\hline & Est. $(95 \% \mathrm{Cl})$ & $\Delta$ Est & P-value & Est. $(95 \% \mathrm{Cl})$ & P-value & Est. $(95 \% \mathrm{CI})$ & P-value \\
\hline Baseline model ${ }^{a}$ & $0.708(0.672-0.744)$ & - & - & - & - & - & - \\
\hline +TyG index & $0.758(0.726-0.791)$ & 0.050 & $<0.001$ & $0.255(0.145-0.320)$ & $<0.001$ & $0.033(0.012-0.058)$ & $<0.001$ \\
\hline$+\mathrm{VAl}$ & $0.734(0.700-0.769)$ & 0.026 & 0.004 & $0.149(0.076-0.244)$ & $<0.001$ & $0.025(0.010-0.046)$ & $<0.001$ \\
\hline+ CVAl & $0.726(0.692-0.761)$ & 0.018 & 0.029 & $0.168(0.033-0.239)$ & 0.007 & $0.008(0.000-0.023)$ & 0.033 \\
\hline$+L A P$ & $0.742(0.707-0.777)$ & 0.034 & $<0.001$ & $0.155(0.060-0.233)$ & $<0.001$ & $0.026(0.009-0.053)$ & $<0.001$ \\
\hline$+\mathrm{TG} / \mathrm{HDL}-\mathrm{C}$ & $0.731(0.696-.0766)$ & 0.023 & $<0.001$ & $0.134(0.054-0.227)$ & $<0.001$ & $0.020(0.006-0.041)$ & $<0.001$ \\
\hline
\end{tabular}

TyG triglyceride-glucose, VAl visceral adiposity index, CVAI Chinese visceral adiposity index, LAP lipid accumulation product, TG/HDL-C triglyceride-to-high density lipoprotein cholesterol ratio, $\mathrm{NRI}$ net reclassification improvement, IDI integrated discrimination improvement, $\mathrm{Cl}$ confidence interval

${ }^{a}$ The baseline model incorporates smoking history, hypertension, T2DM, previous MI, previous PCl, previous stroke, TC, eGFR, HbA1C, LVEF, LM disease, three-vessel disease, SYNTAX score, and number of stents 
unanimous in the subgroup analysis where similar analyses were performed in patients with and without T2DM, respectively.

\section{Clinical significance and quantification of IR}

IR, which is characterized as decreasing efficiency and compensatory secretion of insulin [9], has been well demonstrated to be closely associated with ASCVD. Since the gold standard method is complex, time-consuming, and expensive, it is of great clinical significance to explore simple surrogate markers for evaluating IR.

The homeostasis model assessment of IR (HOMA-IR), which is determined by FBG and fasting insulin, the two most important elements of IR, has been regarded as the generally accepted surrogate marker of IR and shown to be closely related to ASCVD [32]. However, the measurement of fasting insulin is not routinely conducted in clinical practice and varies between different laboratories, especially for non-diabetic patients, which makes HOMA-IR unsuitable for widespread clinical application. Besides increased FBG and fasting insulin, IR has been also proved to be characterized as dyslipidemia (especially increased fasting TG and decreased HDL-C), and visceral obesity $[6,9]$. On account of these characteristics, various indicators originated from conventional laboratory and anthropometric indices have been proposed as substitutes for assessing the level of IR. Unlike the HIEG clamp and HOMA-IR, which are complicated, time and cost consuming, and insulin-dependent, IR surrogates including the TyG index, VAI, CVAI, LAP, and TG/HDL-C exhibit the superiority of simplicity, accessibility, inexpensiveness, and insulin-independence, indicating the great potential of them to be widely used in clinic as valuable indicators reflecting the level of IR.

\section{Associations between IR surrogates and ASCVD}

IR surrogates have been proved to be closely related to the development and progression of ASCVD in subsequent studies. Irace et al. [19] found that the TyG index exhibited a higher correlation than HOMA-IR with carotid atherosclerosis, suggesting that the TyG index could better reflect the cardiovascular risk. The study of Cho et al. [33] showed that elevated TyG index and TG/ HDL-C were independently correlated with an increased risk of CAD. However, the correlation was only significant in non-diabetic patients, and HOMA-IR did not show a predictive value for CAD in this study. The association of the TyG index with the risk of developing ASCVD has also been verified by a series of studies, in both diabetic and non-diabetic population [20, 34-36]. The ATTICA study [21, 22] revealed that VAI and LAP were independently associated with long-term risk of ASCVD, indicating that VAI and LAP could be useful predictors for identifying individuals at high risk of ASCVD in the general population. Findings from Da silva et al. [23] further revealed that increased TyG index was significantly associated with a higher prevalence of symptomatic CAD in patients in secondary care. In addition, for patients with pre-existing ASCVD, IR surrogates including the TyG index and TG/HDL-C have been shown to be significantly associated with the risk of adverse prognosis [24-26, 37-39].

However, there is a relative lack of researches aiming at investigating and comparing the value of various IR surrogates on the prediction and stratification for the risk of adverse prognosis following PCI in patients diagnosed with NSTE-ACS. The present study, which confirmed the prognostic impact of various IR surrogates and further identified the superiority of the TyG index over other IR surrogates on the prediction and stratification for the risk of MACCE, fills in the gaps of previous studies in the comparison of the prognostic value of different IR surrogates. Moreover, the superiority of the TyG index on risk prediction and stratification was shown to be consistent in both subgroups with and without T2DM, suggesting that the TyG index could be served as the most valuable IR surrogate providing more information on adverse prognosis, independent of the presence of T2DM.

\section{Potential explanations for the superiority of the TyG index on the prognostic value}

As described above, IR is usually characterized as increased FBG, fasting insulin, and fasting TG, decreased HDL-C, central obesity (especially increased visceral fat), and so forth, but the roles and importance they played in the quantification of IR have not been fully investigated. IR surrogates derived from different combinations of the characteristics mentioned above may reflect the level of IR from various aspects. Former study has shown that fasting TG mainly reflects IR from the adipose tissue, whereas FBG mainly reflects IR from the liver [40]. Therefore, it can be easily generalized that the TyG index, which is calculated from fasting TG and FBG, may reflect IR from the two most significant dimensions, thus making it more closely associate with the level of IR. This may be an important part of the explanation for the results of the present study that the TyG index, in contrast to other IR surrogates, plays the most pronounced role in prediction and stratification for the risk of adverse outcomes.

In addition, a large number of studies have shown that the TyG index is closely related to vascular calcification [41-43], and arterial stiffness assessed by pulse wave velocity $[44,45]$, both of which were important risk predictors for ASCVD. Moreover, the association between the TyG index and a variety of risk factors for ASCVD, for example, hypertension [46], renal dysfunction [47], 
and hyperuricemia [48], was also confirmed by previous studies. As for other IR surrogates, however, the corresponding evidence was relatively lacking, which may be another important explanation for the superiority of the TyG index.

\section{Mechanisms mediating the associations between IR surrogates and ASCVD}

Since the high correlation between IR surrogates and the HIEG clamp, the gold standard method for evaluating the IR levels, has been well established by former studies $[10,14-16]$, the close relationship of IR surrogates with ASCVD may be mainly mediated by IR, which promotes the development and progression of atherosclerosis through various mechanisms. It has been widely illustrated that IR is closely associated with endothelial dysfunction, oxidative stress, smooth muscle cell proliferation and migration, inflammatory response, and endothelin-1 overproduction $[6,49,50]$, all of which have been considered to be significant pathogenesis for the formation and progression of atherosclerosis. Furthermore, IR has been also revealed to be highly correlated with cardiovascular remodeling, myocardial hypoperfusion, microcirculatory dysfunction, and thrombosis imbalance [51-53], which may be the potential explanations for the significant predictive value of IR surrogates for adverse prognosis.

\section{Study strengths and limitations}

The current study, which investigated the predictive value of various IR surrogates for adverse prognosis in patients with NSTE-ACS who were treated with PCI, remarkably found that the TyG index showed exceptional performance beyond other IR surrogates on risk prediction and stratification in this selected high-risk population. More importantly, the superiority of the TyG index remained consistent regardless of the presence of T2DM. To our knowledge, this is the first study comprehensively exploring the superiority of different IR surrogates on risk prediction and stratification for adverse prognosis following PCI in patients with NSTE-ACS.

However, some limitations listed as follows need to be noted. (1) As a single-center, observational cohort study with a relatively shorter follow-up time, the statistical power may be limited. (2) The study population was highly selected with strict enrollment criteria, which may result in selection bias and difficulties in generalizing the findings to other populations. (3) Despite dynamic changes [54] and the mean of multiple monitoring [55] of the TyG index during the follow-up having been proved to be closely associated with cardiovascular events, they were not accessible in the current analysis. Further group-based trajectory analysis is needed to provide more reliable findings. (4) Lipidlowering and antidiabetic therapy, though adjusted and/or alleviated in analysis, may have an underlying impact on the study results. (5) The diet characteristics, which may have a great influence on the estimation of IR surrogates, were not available in the present study. (6) Although blood samples were obtained at a fasting time of $\geq 8 \mathrm{~h}$, the definite fasting duration was not accessible, which may have a potential effect on the results. (7) The gold standard and generally admitted methods for evaluating IR, HIEG clamp and HOMAIR, were not attainable in this study, which makes the comparison between the existing IR surrogates and them unavailable.

\section{Conclusion}

Compared with other IR surrogates, the TyG index is most strongly associated with the risk of MACCE in NSTE-ACS patients who received PCI, either in those with or without T2DM. The TyG index, which is derived from fasting TG and FBG, may provide more valuable information than other IR surrogates in identifying patients at high risk of developing adverse prognosis in this selected population.

\begin{abstract}
Abbreviations
IR: Insulin resistance; T2DM: Type 2 diabetes mellitus; ASCVD: Atherosclerotic cardiovascular disease; HIEG: Hyperinsulinaemic-euglycaemic; TyG: Triglyceride-glucose; VAl: Visceral adiposity index; CVAl: Chinese visceral adiposity index; LAP: Lipid accumulation product; TG/HDL-C: Triglyceride-to-high density lipoprotein cholesterol ratio; PCl: Percutaneous coronary intervention; NSTE-ACS: Non-ST-segment elevation acute coronary syndrome; NSTEMI: Non-ST-segment elevation myocardial infarction; UA: Unstable angina; BMI: Body mass index; WC: Waist circumference; CAD: Coronary artery disease; MI: Myocardial infarction; PAD: Peripheral artery disease; TG: Triglyceride; TC: Total cholesterol; LDL-C: Low-density lipoprotein cholesterol; HDL-C: High-density lipoprotein cholesterol; hs-CRP: High-sensitivity C-reactive protein; eGFR: Estimated glomerular filtration rate; FBG: Fasting blood glucose; $\mathrm{HbA} 1 \mathrm{c}$ : Glycosylated hemoglobin A1c; LVEF: Left ventricular ejection fraction; ACEl: Angiotensin-converting enzyme inhibitor; ARB: Angiotensin receptor blocker; SYNTAX: The synergy between PCI with taxus and cardiac surgery; MACCE: Major cardiovascular and cerebrovascular events; LM: Left main artery; HR: Hazard ratio; Cl: Confidence interval; ROC: Receiver operating characteristics; AUC: Area under the ROC curve; NRI: Net reclassification improvement; IDI: Integrated discrimination improvement; HOMA-IR: Homeostasis model assessment of insulin resistance.
\end{abstract}

\section{Supplementary Information}

The online version contains supplementary material available at https://doi. org/10.1186/s12933-021-01383-7.

Additional file 1. Additional Tables.

Additional file 2. Additional Figures.

Acknowledgements

Not applicable. 


\section{Authors' contributions}

QZ made substantial contributions to study design, data analysis, and manuscript writing. Y-JZ made substantial contributions to study design, intellectual direction, and manuscript revision. Y-JC, Y-KX, Z-WZ, CL, and T-NS made substantial contributions to data collection and follow-up. All authors read and approved the final manuscript.

\section{Funding}

This work was supported by the grant from National Key Research and Development Program of China (2017YFC0908800); Beijing Municipal Administration of Hospitals "Mission plan" (SML20180601); Capital's Funds for Health Improvement and Research (CFH2020-2-2063); KM200910025012; Beijing Municipal Natural Science Foundation (7202041).

\section{Availability of data and materials}

The datasets used and/or analyzed during the present study are available from the corresponding author on reasonable request.

\section{Declarations}

\section{Ethics approval and consent to participate}

The study protocol was endorsed by the Clinical Research Ethics Committee of Beijing Anzhen Hospital, Capital Medical University. All subjects were informed and agreed to participate in the present study.

\section{Consent for publication}

Not applicable.

\section{Competing interests}

The authors declare that they have no competing interests.

Received: 23 July 2021 Accepted: 8 September 2021

Published online: 18 September 2021

\section{References}

1. Bonora E, Kiechl S, Willeit J, Oberhollenzer F, Egger G, Meigs JB, et al. Insulin resistance as estimated by homeostasis model assessment predicts incident symptomatic cardiovascular disease in Caucasian subjects from the general population: the Bruneck study. Diabetes Care. 2007;30(2):318-24.

2. Jeppesen J, Hansen TW, Rasmussen S, Ibsen H, Torp-Pedersen C, Madsbad S. Insulin resistance, the metabolic syndrome, and risk of incident cardiovascular disease: a population-based study. J Am Coll Cardiol. 2007:49(21):2112-9.

3. Iguchi T, Hasegawa T, Otsuka K, Matsumoto K, Yamazaki T, Nishimura S, et al. Insulin resistance is associated with coronary plaque vulnerability: insight from optical coherence tomography analysis. Eur Heart J Cardiovasc Imaging. 2014;15(3):284-91.

4. Uetani T, Amano T, Harada K, Kitagawa K, Kunimura A, Shimbo Y, et al. Impact of insulin resistance on post-procedural myocardial injury and clinical outcomes in patients who underwent elective coronary interventions with drug-eluting stents. JACC Cardiovasc Interv. 2012;5(11):1159-67.

5. Gast KB, Tjeerdema N, Stijnen T, Smit JW, Dekkers OM. Insulin resistance and risk of incident cardiovascular events in adults without diabetes: meta-analysis. Plos One. 2012;7(12):e52036.

6. Ormazabal V, Nair S, Elfeky O, Aguayo C, Salomon C, Zuñiga FA. Association between insulin resistance and the development of cardiovascular disease. Cardiovasc Diabetol. 2018;17(1):122

7. Zethelius B, Lithell H, Hales CN, Berne C. Insulin sensitivity, proinsulin and insulin as predictors of coronary heart disease. A population-based 10-year, follow-up study in 70-year old men using the euglycaemic insulin clamp. Diabetologia. 2005;48(5):862-7.

8. Wiberg B, Sundström J, Zethelius B, Lind L. Insulin sensitivity measured by the euglycaemic insulin clamp and proinsulin levels as predictors of stroke in elderly men. Diabetologia. 2009;52(1):90-6.

9. Di Pino A, DeFronzo RA. Insulin resistance and atherosclerosis: implications for insulin-sensitizing sgents. Endocr Rev. 2019;40(6):1447-67.
10. Guerrero-Romero F, Simental-Mendía LE, González-Ortiz M, MartínezAbundis E, Ramos-Zavala MG, Hernández-González SO, et al. The product of triglycerides and glucose, a simple measure of insulin sensitivity. Comparison with the euglycemic-hyperinsulinemic clamp. J Clin Endocrinol Metab. 2010;95(7):3347-51.

11. Amato MC, Giordano C, Galia M, Criscimanna A, Vitabile S, Midiri M, et al. Visceral Adiposity Index: a reliable indicator of visceral fat function associated with cardiometabolic risk. Diabetes Care. 2010;33(4):920-2.

12. Xia MF, Chen Y, Lin HD, Ma H, Li XM, Aleteng $Q$, et al. A indicator of visceral adipose dysfunction to evaluate metabolic health in adult Chinese. Sci Rep. 2016;6:38214.

13. Kahn HS. The "lipid accumulation product" performs better than the body mass index for recognizing cardiovascular risk: a population-based comparison. BMC Cardiovasc Disord. 2005;5:26.

14. McLaughlin T, Reaven G, Abbasi F, Lamendola C, Saad M, Waters D, et al. Is there a simple way to identify insulin-resistant individuals at increased risk of cardiovascular disease? Am J Cardiol. 2005;96(3):399-404.

15. Fiorentino TV, Marini MA, Succurro E, Andreozzi F, Sesti G. Relationships of surrogate indexes of insulin resistance with insulin sensitivity assessed by euglycemic hyperinsulinemic clamp and subclinical vascular damage. BMJ Open Diabetes Res Care. 2019;7(1):e911.

16. Du T, Yuan G, Zhang M, Zhou X, Sun X, Yu X. Clinical usefulness of lipid ratios, visceral adiposity indicators, and the triglycerides and glucose index as risk markers of insulin resistance. Cardiovasc Diabetol. 2014;13:146.

17. Ahn N, Baumeister SE, Amann U, Rathmann W, Peters A, Huth C, et al. Visceral adiposity index (VAI), lipid accumulation product (LAP), and product of triglycerides and glucose (TyG) to discriminate prediabetes and diabetes. Sci Rep. 2019;9(1):9693.

18. Zhang M, Wang B, Liu Y, Sun X, Luo X, Wang C, et al. Cumulative increased risk of incident type 2 diabetes mellitus with increasing triglyceride glucose index in normal-weight people: the rural Chinese cohort study. Cardiovasc Diabetol. 2017;16(1):30.

19. Irace C, Carallo C, Scavelli FB, De Franceschi MS, Esposito T, Tripolino C, et al. Markers of insulin resistance and carotid atherosclerosis. A comparison of the homeostasis model assessment and triglyceride glucose index. Int J Clin Pract. 2013;67(7):665-72.

20. Park G, Cho Y, Won K, Yang YJ, Park S, Ann SH, et al. Triglyceride glucose index is a useful marker for predicting subclinical coronary artery disease in the absence of traditional risk factors. Lipids Health Dis. 2020;19(1):7.

21. Kouli GM, Panagiotakos DB, Kyrou I, Georgousopoulou EN, Chrysohoou C, Tsigos C, et al. Visceral adiposity index and 10-year cardiovascular disease incidence: the ATTICA study. Nutr Metab Cardiovasc Dis. 2017;27(10):881-9.

22. Kyrou I, Panagiotakos DB, Kouli GM, Georgousopoulou E, Chrysohoou C, Tsigos C, et al. Lipid accumulation product in relation to 10-year cardiovascular disease incidence in Caucasian adults: the ATTICA study. Atherosclerosis. 2018;279:10-6.

23. Da Silva A, Caldas APS, Hermsdorff HHM, Bersch-Ferreira ÂC, Torreglosa $C R$, Weber $B$, et al. Triglyceride-glucose index is associated with symptomatic coronary artery disease in patients in secondary care. Cardiovasc Diabetol. 2019;18(1):89.

24. Yang SH, Du Y, Li XL, Zhang Y, Li S, Xu RX, et al. Triglyceride to high-density lipoprotein cholesterol ratio and cardiovascular events in diabetics with coronary artery disease. Am J Med Sci. 2017;354(2):117-24.

25. Wang L, Cong HL, Zhang JX, Hu YC, Wei A, Zhang YY, et al. Triglycerideglucose index predicts adverse cardiovascular events in patients with diabetes and acute coronary syndrome. Cardiovasc Diabetol. 2020;19(1):80.

26. Luo E, Wang D, Yan G, Qiao Y, Liu B, Hou J, et al. High triglyceride-glucose index is associated with poor prognosis in patients with acute STelevation myocardial infarction after percutaneous coronary intervention. Cardiovasc Diabetol. 2019;18(1):150.

27. Roffi M, Patrono C, Collet J, Mueller C, Valgimigli M, Andreotti F, et al. 2015 ESC Guidelines for the management of acute coronary syndromes in patients presenting without persistent ST-segment elevation. Eur Heart J. 2016:37(3):267-315

28. American Diabetes Association. Diagnosis and classification of diabetes mellitus. Diabetes Care. 2014;37(Suppl 1):S81-90.

29. Section of Interventional Cardiology of Chinese Society of Cardiology of Chinese Medical Association, Specialty Committee on Prevention and Treatment of Thrombosis of Chinese College of Cardiovascular Physicians, 
Editorial Board of Chinese Journal of Cardiology. Chinese guideline for percutaneous coronary intervention (2016). Zhonghua Xin Xue Guan Bing Za Zhi. 2016; 44(5):382-400.

30. Ryan TJ, Faxon DP, Gunnar RM, Kennedy JW, King SR, Loop FD, et al. Guidelines for percutaneous transluminal coronary angioplasty. A report of the American College of Cardiology/American Heart Association Task Force on Assessment of Diagnostic and Therapeutic Cardiovascular Procedures (Subcommittee on Percutaneous Transluminal Coronary Angioplasty). Circulation. 1988;78(2):486-502.

31. Lee J, Kim B, Kim W, Ahn C, Choi HY, Kim JG, et al. Lipid indices as simple and clinically useful surrogate markers for insulin resistance in the U.S. population. Sci Rep. 2021;11(1):2366.

32. Bonora E, Formentini G, Calcaterra F, Lombardi S, Marini F, Zenari L, et al. HOMA-estimated insulin resistance is an independent predictor of cardiovascular disease in type 2 diabetic subjects: prospective data from the Verona Diabetes Complications Study. Diabetes Care. 2002;25(7):1135-41.

33. Cho Y, Ann SH, Won K, Park G, Kim Y, Yang DH, et al. Association between insulin resistance, hyperglycemia, and coronary artery disease according to the presence of diabetes. Sci Rep-Uk. 2019;9(1):6129.

34. Shi W, Xing L, Jing L, Tian Y, Yan H, Sun Q, et al. Value of triglycerideglucose index for the estimation of ischemic stroke risk: insights from a general population. Nutr Metab Cardiovasc Dis. 2020;30(2):245-53.

35. Lee EY, Yang HK, Lee J, Kang B, Yang Y, Lee S, et al. Triglyceride glucose index, a marker of insulin resistance, is associated with coronary artery stenosis in asymptomatic subjects with type 2 diabetes. Lipids Health Dis. 2016;15(1):155.

36. Su W, Chen S, Huang Y, Huang J, Wu P, Hsu W, et al. Comparison of the effects of fasting glucose, hemoglobin A1c, and triglyceride-glucose index on cardiovascular events in type 2 diabetes mellitus. Nutrients. 2019;11(11):2838.

37. Jin JL, Sun D, Cao YX, Guo YL, Wu NQ, Zhu CG, et al. Triglyceride glucose and haemoglobin glycation index for predicting outcomes in diabetes patients with new-onset, stable coronary artery disease: a nested casecontrol study. Ann Med. 2018;50(7):576-86.

38. Sultani R, Tong DC, Peverelle M, Lee YS, Baradi A, Wilson AM. Elevated triglycerides to high-density lipoprotein cholesterol (TG/HDL-C) ratio predicts long-term mortality in high-risk patients. Heart Lung Circ. 2020;29(3):414-21.

39. Deng QW, Li S, Wang H, Lei L, Zhang HQ, Gu ZT, et al. The short-term prognostic value of the triglyceride-to-high-density lipoprotein cholesterol ratio in acute ischemic stroke. Aging Dis. 2018;9(3):498-506.

40. Low S, Khoo K, Irwan B, Sum CF, Subramaniam T, Lim SC, et al. The role of triglyceride glucose index in development of type 2 diabetes mellitus. Diabetes Res Clin Pract. 2018;143:43-9.

41. Chen Y, Chang Z, Zhao Y, Liu Y, Fu J, Zhang Y, et al. Association between the triglyceride-glucose index and abdominal aortic calcification in adults: a cross-sectional study. Nutr Metab Cardiovasc Dis. 2021;31(7):2068-76.

42. Park K, Ahn CW, Lee SB, Kang S, Nam JS, Lee BK, et al. Elevated TyG index predicts progression of coronary artery calcification. Diabetes Care. 2019:42(8):1569-73.
43. Won K, Park EJ, Han D, Lee JH, Choi S, Chun EJ, et al. Triglyceride glucose index is an independent predictor for the progression of coronary artery calcification in the absence of heavy coronary artery calcification at baseline. Cardiovasc Diabetol. 2020;19(1):34.

44. Wu S, Xu L, Wu M, Chen S, Wang Y, Tian Y. Association between triglyceride-glucose index and risk of arterial stiffness: a cohort study. Cardiovasc Diabetol. 2021:20(1):146.

45. Wang S, Shi J, Peng Y, Fang Q, Mu Q, Gu W, et al. Stronger association of triglyceride glucose index than the HOMA-IR with arterial stiffness in patients with type 2 diabetes: a real-world single-centre study. Cardiovasc Diabetol. 2021;20(1):82.

46. Zheng R, Mao Y. Triglyceride and glucose (TyG) index as a predictor of incident hypertension: a 9-year longitudinal population-based study. Lipids Health Dis. 2017;16(1):175.

47. Fritz J, Brozek W, Concin H, Nagel G, Kerschbaum J, Lhotta K, et al. The triglyceride-glucose index and obesity-related risk of end-stage kidney disease in Austrian adults. JAMA Netw Open. 2021;4(3):e212612.

48. Mazidi M, Katsiki N, Mikhailidis DP, Banach M. The link between insulin resistance parameters and serum uric acid is mediated by adiposity. Atherosclerosis. 2018;270:180-6.

49. DeFronzo RA. Insulin resistance, lipotoxicity, type 2 diabetes and atherosclerosis: the missing links. The Claude Bernard Lecture 2009. Diabetologia. 2010;53(7):1270-87.

50. Wilkes JJ, Hevener A, Olefsky J. Chronic endothelin-1 treatment leads to insulin resistance in vivo. Diabetes. 2003:52(8):1904-9.

51. Markus M, Rospleszcz S, Ittermann T, Baumeister SE, Schipf S, SiewertMarkus U, et al. Glucose and insulin levels are associated with arterial stiffness and concentric remodeling of the heart. Cardiovasc Diabetol. 2019;18(1):145.

52. Trifunovic D, Stankovic S, Sobic-Saranovic D, Marinkovic J, Petrovic M, Orlic $\mathrm{D}$, et al. Acute insulin resistance in ST-segment elevation myocardial infarction in non-diabetic patients is associated with incomplete myocardial reperfusion and impaired coronary microcirculatory function. Cardiovasc Diabetol. 2014;13:73.

53. Laakso M, Kuusisto J. Insulin resistance and hyperglycaemia in cardiovascular disease development. Nat Rev Endocrinol. 2014;10(5):293-302.

54. Wang A, Tian X, Zuo Y, Chen S, Meng X, Wu S, et al. Change in triglyceride-glucose index predicts the risk of cardiovascular disease in the general population: a prospective cohort study. Cardiovasc Diabetol. 2021;20(1):113.

55. Tian X, Zuo Y, Chen S, Liu Q, Tao B, Wu S, et al. Triglyceride-glucose index is associated with the risk of myocardial infarction: an 11-year prospective study in the Kailuan cohort. Cardiovasc Diabetol. 2021;20(1):19.

\section{Publisher's Note}

Springer Nature remains neutral with regard to jurisdictional claims in published maps and institutional affiliations.

\footnotetext{
Ready to submit your research? Choose BMC and benefit from:

- fast, convenient online submission

- thorough peer review by experienced researchers in your field

- rapid publication on acceptance

- support for research data, including large and complex data types

- gold Open Access which fosters wider collaboration and increased citations

- maximum visibility for your research: over $100 \mathrm{M}$ website views per year
}

At BMC, research is always in progress.

Learn more biomedcentral.com/submissions 